\title{
Reduction of emotional responses as a function of verbal satiation and paired-associate techniques*
}

\author{
NICHOLAS S. DiCAPRIO \\ John Carroll University, Cleveland, Ohio 44118
}

\begin{abstract}
Verbal satiation of emotional words was combined with paired-associate procedures to reduce the subjective and physiological response intensity. Satiation effects occurred with the verbal satiation techniques alone and were further enhanced by the associative procedures. The emotional words were paired with counteracting emotional response associates (CRA group), and with personally provided response associates (PRA group). The types of associates did not produce different results. Counteracting associates appeared to yield the best long-term satiation effects.
\end{abstract}

Rapidly repeating a word aloud for a minute or more tends to alter its stimulus properties. Its symbolic character undergoes modification with treatment, and both the cognitive and affective aspects are altered. This phenomenon has been called verbal satiation. If one repeats a highly emotionally charged word for a short period of time, a gradual reduction in GSR will occur. If this process is repeated several times (DiCaprio \& Turnock, 1969), GSR levels will approach baseline. With repeated satiation, the lowered GSR will persist for from a week to several months.

Specific cognitive aspects which are altered by satiation include the following: The latency of response to satiated words is slower than to nonsatiated ones (Baras, 1968); satiated words yield fewer associations in a given period of time than do nonsatiated words (Fillenbaum, 1963; Smith \& Raygor, 1956); associations given to satiated words are rarer, as measured by frequency tables such as the Jenkins-Palermo frequency tables (Baras, 1968; Fillenbaum, 1963; Noble, 1952); the learning of associations has been demonstrated to be hindered when stimuli are satiated and when the responses are satiated (Gorfein, 1967); if highly meaningful words are satiated before being learned as paired associates, the learning is significantly slower than that of similar, but nonsatiated, pairs (Smith \& Raygor, 1956).

This study investigated other techniques for producing the satiation effects, specifically, associative learning in which (a) the paired responses have a counteracting positive affective tone, a condition designated "counteracting response associates" (CRA); or, (b) have an unusual connection with the emotional words, a condition called "personally provided response associates" (PRA). A second purpose was to find techniques for prolonging the satiation effects. The success of clinical application of satiation therapy

\footnotetext{
*Special thanks are due to William S. Lavezzi for his substantial assistance in the preparation of this report.
}

depends both upon the degree of satiation that can be produced and the persistence of its effects.

\section{METHOD}

Sixty students in a freshman psychology class were divided into three groups. The members of the control group were subjected to the paired-associate task only, not to the satiation procedure. whereas the two experimental groups received both.

\section{Pretest Phase}

The $S$ came into the experimental room with 15 emotionally charged words, which had been rated 2 or more weeks earlier, and 20 neutral words. After brief instructions, GSR electrodes were attached and a short warm-up period with the GSR machine running was begun, to permit the $S$ to become accustomed to the situation. The $\mathrm{S}$ was then asked to say each of the 15 emotional words slowly three times. Neutral words were interspersed among the emotional words to bring the response level back to baseline. The E selected 6 of the most emotional words, defined as those which produced GSR responses at least $19.5 \mathrm{~cm}$ above baseline. This requirement was used to eliminate Ss who were not cooperating with the experiment; 15 of the original $60 \mathrm{Ss}$ were excluded for this reason, leaving three groups of $15 \mathrm{Ss}$ each. The 6 selected words were then written on cards by the $\mathrm{E}$. The $\mathrm{S}$ was presented one card at a time, was asked to fixate it, and then was told to repeat the word slowly three times. The GSR response for these 6 words constituted the pretest measure for GSR. Rank sorting consisted of Ss sorting the cards according to the emotional intensity of each word.

\section{Satiation Phase}

The satiation phase required the $S$ to satiate an emotional word by rapid repetition for $60 \mathrm{sec}$, followed by several repetitions of a neutral word. Then another emotional word was satiated for $60 \mathrm{sec}$, and again a neutral word was repeated several times. The $S$ went through all 6 selected emotionally charges words in this manner. Following a rest period of $2.3 \mathrm{~min}$, the whole procedure was repeated. Thus, each emotional word received 2 min of satiation treatment.

Reaction times were taken twice during the satiation phase: during the first minute of satiation for each word and during the second minute of satiation. Reaction time measured time lapses required for a subjective change in the words' stimulus properties. Ss simply depressed a key at the first instance of a subjective change.

Satiation sessions were not given to the control group. These Ss simply filled out a questionnaire during this period.

\section{Posttest Phase}

The GSR posttest was taken immediately after the satiation treatment in exactly the same manner as the pretest phase.

The learning phase followed the satiation phase. All three groups participated in it. For the PRA group, however, it was first necessary to obtain the S's own associations. This was done immediately after the GSR test for satiation, by presenting the satiated emotional words one at a time and requesting the appropriate number of associations ( 1 to 5 ).

\section{Final Test Phase}

All groups were treated identically for the association task. Each emotional word was presented, following which each $\mathrm{S}$ was given from 1 to 5 associations to pair with it. The control and one of the experimental groups were given the CRA words, and the other experimental group the PRA words. After the 
Table 1

Schema of Research Design

\begin{tabular}{lcccc}
\hline Experimental Operation and Groups & Control CRA & PRA \\
\hline Initial Phase & & & \\
Obtaining of Anxiety Words & + & + & + \\
Pretests & & & \\
Subjective Ratings & + & + & + \\
Rank Sorting & + & + & + \\
GSR & + & + & + \\
Satiation & Omit & + & + \\
Interim & Omit & + & + \\
GSR Posttest & & + & \\
Obtaining of Personally Provided Associates & Omit & Omit & + \\
Providing Counteracting Associates & + & + & Omit \\
Association & + & + & + \\
Testing & & & \\
GSR and Rank Sorting Final Tests & + & + & + \\
Subjective Ratings Posttest & + & + & + \\
Followup: Subjective Ratings Final Test & + & + & + \\
\hline
\end{tabular}

association phase, the words were rerated on the 0-100 scale. This was followed by the final GSR test, and this in turn was followed by a resorting of the words according to rank order. The total test time was about $90 \mathrm{~min}$.

\section{Followup Phase}

Three to 6 months after the experiment, the Ss were sent a letter requesting them to list 15 emotionally charged words and to rate them on the 0 -to- 100 scale. This constituted the final test for the subjective ratings measure. The entire procedure is presented schematically in Table 1 .

\section{RESULTS \\ Reaction Time}

Analysis of the reaction time data indicates that no significant change in reaction time occurred between the satiation treatments for either the CRA or the PRA group. Reaction time is not a valid measure of satiation effects.

\section{Rank Sorting}

The prefinal card-sorting values did not attain significance, nor is there any consistent pattern evident in regard to the number of associative responses per emotional word.

\section{Subjective Ratings}

Analysis of the subjective ratings suggest that no significant reduction in emotional responses had occurred for the three groups as the result of treatments. The prepost subjective rating comparisons are as follows: control group $(\mathrm{N}=90), \mathrm{t}=.75 ;$ CRA group $(\mathrm{N}=90), \mathrm{t}=$ $.75 ;$ PRA group $(\mathrm{N}=90), \mathrm{t}=.52$.

With the exception of the five-associate condition for the control group and the one-associate condition for the PRA group $(\mathrm{N}=15, \mathrm{t}=5.2, \mathrm{p}<.005 ;$ and $\mathrm{N}=15, \mathrm{t}$ $=2.8, p<.05$, respectively), the number of associates was not a significant variable for a subjective reduction in emotional responses immediately after treatment.

With respect to the CRA and PRA groups, there was no significant difference between the post and follow-up ratings, although it is noteworthy that the follow-up ratings were somewhat lower than they were immediately after treatments.

A key finding of this study was that after 3 to 6 months, the control group experienced a significant reduction in subjective ratings of emotional words $(\mathrm{N}=$ $40, t=2.4, p<.05$ ), whereas the CRA and PRA groups did not.

\section{GSR Analysis}

Significant emotional reduction occurred for all three groups. The $\mathrm{Ss}$ in the control group experienced a significant reduction in emotional level $(39.7 \%, \mathrm{~N}=90, \mathrm{t}$ $=3.7, \mathrm{p}<.005)$. GSR pretest scores dropped significantly following the associative learning, as measured by the GSR posttest for all numbers of associates from 0 to 5 , and for the group as a whole. It should be noted that the control group was not subjected to satiation. This drop in response intensity was contrary to expectations.

The $t$ test comparisons for the CRA and PRA groups indicated that a significant reduction in GSR levels occurred for the pre- and post-satiation measures (CRA group: $\mathrm{N}=90, \mathrm{t}=7.1, \mathrm{p}<.005$; PRA group: $\mathrm{N}=90, \mathrm{t}=$ $7.2, p<.001)$. A further reduction in GSR levels occurred between the post and final GSR measure as the result of the associative learning treatment for the CRA and PRA groups (CRA: $34.6 \%, \mathrm{~N}=90, \mathrm{t}=2.8$, $\mathrm{p}<.005$; PRA: $36.5 \%, \mathrm{~N}=90, \mathrm{t}=3.5, \mathrm{p}<.005$ ).

There was a significant pretest to final GSR reduction of $70.7 \%(\mathrm{~N}=90, \mathrm{t}=8.7, \mathrm{p}<.005)$ for the CRA group, and a reduction of $73.0 \%(\mathrm{~N}=90, t=9.9, p<.005)$ for the PRA group. It may be concluded that the satiation procedure was effective in reducing emotional responses as measured by GSR, and the association procedure was also effective. Furthermore, the emotional level after satiation could be lowered even more with the application of association learning.

The number of associative responses for each emotional word, which varied from 0 to 5 , did not yield decreasing emotional intensity as a function of increasing numbers of associations. Two significant points should be noted: First, the optimum reduction in emotional intensity occurred with one association $(\mathrm{N}=$ $45, \mathrm{t}=5.0, \mathrm{p}<.001)$; second, the zero association condition (anxiety words which received no associative learning) also yielded a significant reduction in emotional intensity $(\mathrm{N}=45, \mathrm{t}=2.8, \mathrm{p}<.005)$.

Because the zero-association condition yielded significant emotional reduction, the possibility of a generalization effect was raised. As a result, the neutral words were analyzed for any reduction in GSR response which might have occurred between pre- and final GSR testing. A "neutral word" was defined as one which did not meet the criterion of a $19.5 \mathrm{~cm}$ GSR response height. Thirty neutral words were selected at random from each of the three groups, and their prefinal GSR levels subjected to $t$ tests. The neutral words for each of the three groups yielded a reduction in GSR levels 
significant at the .05 level. The conclusion of a generalized emotional reduction as a result of the treatments is thus supported.

\section{DISCUSSION}

One of the most significant findings of the study is that the CRA procedure with the control group produced satiation of emotional words. All measures of response intensity were lower for the control group on posttest. Notably, only the posttest GSR level (39.7\% reduction in emotional intensity) attained significance. Furthermore, the subjective ratings for the control group 3 to 6 months later were the lowest of the three groups studied. The data suggest that the CRA procedure alone yields long-term emotional reduction.

With respect to the number of CRAs for each emotional word, the most consistent finding is that a single CRA yields optimal reduction of emotional intensity, though additional exploration of this effect in the clinic is necessary.

Two minutes of verbal repetition yielded the expected satiation effects. Satiation was further enhanced by the addition of either the CRA or PRA procedures. It may be, however, that the CRA procedure can take the place of verbal repetition and that, in fact, the CRA procedure is a form of verbal satiation. When verbal repetition and CRA procedures were used consecutively. the satiation effects were greater than either procedure produced alone, as measured by GSR tracings.

Whether the CRAs or PRAs made better associates was not settled, although the PRA group yielded slightly better reduction of GSR levels. It appears that the associative procedure itself was sufficient to produce satiation effects.

All of the words that were tested, including those that did not receive treatment (the zero association condition), were significantly lower in intensity on retest. It should also be noted, though, that the neutral words were also reduced in intensity. Thus, a general reduction in emotional level affected all the words, neutral as well as untreated. This conclusion is consistent with the generalized effects of symptom alleviation, in that the elimination or reduction of a symptom reduces the intensity of other symptoms.

All three groups had lowered subjective ratings on follow-up, although the ratings of only the control group attained statistical significance. These data, taken at face value, suggest that subjective reduction of emotional level lags behind physiological reduction. Two questions prompted by the results of the study are: Why did only the control group attain significant lowering of ratings on the follow-up? Why should the effects persist with only one treatment session?

\section{REFERENCES}

Baras, H. L. Effect of mode of presentation on semantic satiation. Psychonomic Science, 1968, 11, 345-346.

DiCaprio, N. S., \& Turnock, M. Persistence of GSR changes as a function of repeated verbal satiation of emotionally-charged words. Psychonomic Science, 1969, 17, 238-239.

Fillenbaum, S. Verbal satiation and changes in meaning of related items. Journal of Verbal Learning \& Verbal Behavior, 1963, 2, 263-271.

Gorfein, D. S. Semantic satiation and word association. Psychonomic Science, 1967, 7, 47-48.

Kanungo, R., \& Lambert, W. E. Paired-associate learning as a function of stimulus and response satiation. Journal of Psychology, 1963, 54, 135-144.

Noble, C. E. An analysis of meaning. Psychological Review, $1952,59,421-430$.

Smith, E. D. P., \& Raygor, A. L. Verbal satiation and personality. Journal of Psychology, 1956, 52, 323-326.

(Received for publication October 10,1973.) 